\title{
ESTIMATES FOR THE CAUCHY MATRIX OF PERTURBED LINEAR IMPULSIVE EQUATION
}

\author{
P. S. SIMEONOV and D. D. BAINOV \\ P.O. Box 45 \\ 1504 Sofia, Bulgaria
}

(Received March 24, 1992)

\begin{abstract}
Estimates for the Cauchy matrix of a perturbed linear impulsive equation are obtained for given estimates for the Cauchy matrix of the corresponding unperturbed linear impulsive equation.
\end{abstract}

KEY WORDS AND PHRASES. Cauchy matrix and perturbed linear impulsive equations. 1991 AMS SUBJECT CLASSIFICATION CODE. 34A39.

\section{INTRODUCTION.}

Consider the linear impulsive equation

$$
\begin{array}{ll}
x^{\prime}=A(t) x, & t \neq \tau_{k}, \\
\Delta x=A_{k} x, & t=\tau_{k},
\end{array}
$$

where $t$ belongs to the interval $J \subset \mathbf{R}: \tau_{k}<\tau_{k+1}(k \in \mathbf{Z})$; the sequence $\left\{\tau_{k}\right\}$ has no finite accumulation point; $x \in \mathbf{R}^{n} \cdot, A_{k} \in \mathbf{R}^{n \times n}$. Suppose that $A(t)$ belongs to the space $P C\left(J, \mathbf{R}^{n \times n}\right)$, i.e. $A(t)$ is an $n \times n$ matrix-valued function which is continuous for $t \in J, t \neq \tau_{k}$, and at the points $\tau_{k} \in J$ it has discontinuities of the first kind and is continuous from the left. We recall [1] that the solution $x(t)$ of $(1.1)$ for $t \in J, t \neq \tau_{k}$ satisfies the equation

$$
x^{\prime}=A(t) x \text { and for } t=\tau_{k}
$$

the conditions

$$
x\left(\tau_{k}^{-}\right) \stackrel{\text { def }}{=} \lim _{t \rightarrow \tau_{k}-0} x(t)=x\left(\tau_{k}\right), x\left(\tau_{k}^{+}\right) \stackrel{\text { def }}{=} \lim _{t \rightarrow \tau_{k}+0} x(t)=x\left(\tau_{k}\right)+\Delta x\left(\tau_{k}\right)=x\left(\tau_{k}\right)+A_{k} x\left(\tau_{k}\right) .
$$

Let $|x|$ be a norm of the vector $x \in \mathbf{R}^{n}$ and $|A|=\sup \{|A x|:|x|=1\}$ be the corresponding norm of the matrix $A \in \mathbf{R}^{n \times n}$. Let the Cauchy matrix $W(t, s)$ of (1.1) satisfy an estimate of the form

$$
|W(t, s)| \leq \varphi(t) \psi(s) \quad(s, t \in J, s \leq t),
$$

where the functions $\varphi, \psi: J \rightarrow \mathbf{R}_{+}$continuous and positive.

Based on estimate (1.2), we shall seek for various estimates for the Cauchy matrix $Q(t, s)$ of the perturbed linear equation

$$
\begin{array}{cc}
y^{\prime}=[A(t)+B(t)] y, & t \neq \tau_{k}, \\
\Delta y=\left[A_{k}+B_{k}\right] y, & t=\tau_{k},
\end{array}
$$


where $B(t) \in P C\left(J, \mathbf{R}^{n \times n}\right)$ and $B_{k} \in \mathbf{R}^{n \times n}$.

We shall use the following lemma:

LEMMA 1.1 [2]. Let the function $u \in P C\left(J, \mathbf{R}_{+}\right)$satisfy the inequality

$$
u(t) \leq c+\int_{s}^{t} p(\tau) u(\tau) d \tau+\sum_{s \leq \tau_{k}<t} p_{k} u\left(\tau_{k}\right) \quad(s, t \in J, s \leq t),
$$

where $c \geq 0$ and $p_{k} \geq 0$ are constants and $p(\tau) \in P C\left(J, \mathbf{R}_{+}\right)$.

Then

$$
u(t) \leq c \prod_{s \leq \tau}<t \quad\left(1+p_{k}\right) \mid \exp \left[\int_{0}^{t} p(\tau) d \tau\right] \quad(s, t \in J, s \leq t) .
$$

\section{MAIN RESULTS.}

Recall [1] that if $U_{k}(t, s)$ is the Cauchy matrix for the equation

$$
x^{\prime}=A(t) x \quad\left(\tau_{k-1}<t \leq \tau_{k}\right),
$$

then the Cauchy matrix for equation (1.1) is

$$
W(t, s)=\left\{\begin{array}{cc}
U_{k}(t, s) & \left(s, t \in\left(\tau_{k-1}, \tau_{k}\right]\right), \\
U_{k+1}\left(t, \tau_{k}^{+}\right)\left(E+A_{k}\right) U_{k}\left(\tau_{k}, s\right) & \left(\tau_{k-1}<s \leq \tau_{k}<t \leq \tau_{k+1}\right), \\
U_{k+1}\left(t, \tau_{k}^{+}\right) \prod_{j=k}^{+1}\left(E+A_{j}\right) U_{j}\left(\tau_{j}, \tau_{j-1}^{+}\right)\left(E+A_{i}\right) U_{i}\left(\tau_{i}, s\right) & \left(\tau_{i-1}<s \leq \tau_{i}<\tau_{k}<t \leq \tau_{k+1}\right) .
\end{array}\right.
$$

Then an arbitrary solution $y(t)$ of (1.3) satisfies the integro-summary equation

$$
y(t)=W(t, s) y(s)+\int_{s}^{t} W(t, \tau) B(\tau) y(\tau) d \tau+\sum_{s \leq \tau_{k}<t} W\left(t, \tau_{k}^{+}\right) B_{k} y\left(\tau_{k}\right) .
$$

From (2.1) and (1.2) it follows that

$$
|y(t)| \leq \varphi(t) \psi(s)|y(s)|+\int_{s}^{t} \varphi(t) \psi(\tau)|B(\tau)||y(\tau)| d \tau+\sum_{s \leq \tau_{k}<t} \varphi(t) \psi\left(\tau_{k}\right)\left|B_{k}\right|\left|y\left(\tau_{k}\right)\right| .
$$

The the function $u(t)=|y(t)| / \varphi(t)$ satisfies the inequality

$$
u(t) \leq \psi(s)|y(s)|+\int_{s}^{t} \varphi(\tau) \psi(\tau)|B(\tau)| u(\tau) d \tau+\sum_{s \leq \tau_{k}<t} \varphi\left(\tau_{k}\right) \psi\left(\tau_{k}\right)\left|B_{k}\right| u\left(\tau_{k}\right) .
$$

We apply Lemma 1.1 and obtain the estimate

$$
|y(t)| \leq|y(s)| M(t, s)
$$

where

$$
M(t, s)=\varphi(t) \psi(s) \prod_{s \leq \tau_{k}<t}\left(1+\varphi\left(\tau_{k}\right) \psi\left(\tau_{k}\right)\left|B_{k}\right|\right) \exp \left(\int_{s}^{t} \varphi(\tau) \psi(\tau)|B(\tau)| d \tau\right) .
$$

From (2.2) and the equality $y(t)=Q(t, s) y(s)$ there follow immediately the subsequent assertions:

THEOREM 2.1. Let the Cauchy matrix $W(t, s)$ of equation (1.1) satisfy estimate (1.2).

Then the Cauchy matrix $Q(t, s)$ of equation (1.3) satisfies the estimate 


$$
|Q(t, s)| \leq M(t, s) \quad(s, t \in J, s \leq t)
$$

where $M(t, s)$ is given by $(2.3)$

COROLLARY 2.1. If

$$
|W(t, s)| \leq K e^{\alpha(t-s)} \quad(s, t \in J, s \leq t)
$$

where $K>1$ and $\alpha$ are constants, then

$$
|Q(t, s)| \leq K e^{\alpha(t-s)} \prod_{s \leq \tau_{k}<t}\left(1+K\left|B_{k}\right|\right) \exp \left[\int_{s}^{t} K|B(\tau)| d \tau\right](s, t \in J, s \leq t) .
$$

COROLLARY 2.2. If in the interval $J=\mathbf{R}_{+}$estimate (2.4) is valid and there exists a constant $\delta>0$ such that

then

$$
\sup _{\tau \in \mathbf{R}_{+}}|B(\tau)| \leq \delta, \quad \sup _{\tau_{k} \in \mathbf{R}_{+}}\left|B_{k}\right| \leq \delta
$$

$$
|Q(t, s)| \leq K e^{\alpha(t-s)} e^{K \delta(t-s)+\ln (1+K \delta) i[s, t)},
$$

where $i[s, t)$ is the number of points $\tau_{k}$ lying in the interval $[s, t)$.

Moreover, if there exist constants $q \geq 0$ and $\varepsilon \geq 0$ such that

$$
i[s, t) \leq q(t-s)+\varepsilon,
$$

then

$$
|Q(t, s)| \leq K(1+K \delta)^{\varepsilon} \exp \{[\alpha+K \delta+q \ln (1+K \delta)](t-s)\} \quad(c \leq s \leq t) .
$$

Taking into account that $\prod_{s \leq \tau_{k}<t}\left(1+K\left|B_{k}\right|\right) \leq \exp \sum_{s \leq \tau_{k}<t} K\left|B_{k}\right|$, we obtain

COROLLARY 2.3. In the interval $J=\mathbf{R}_{+}$let estimate (2.4) be valid and let a constant $M>0$ exist such that

$$
\int_{0}^{\infty}|B(\tau)| d \tau+\sum_{\tau_{k} \geq 0}\left|B_{k}\right| \leq M
$$

Then

$$
|Q(t, s)| \leq K e^{K M} \cdot e^{\alpha(t-s)} \quad(0 \leq s \leq t) .
$$

REMARK 1. If equation (1.1) is uniformly asymptotically stable, i.e., estimate (2.4) is valid with $\alpha<0$, then under perturbations for which (2.6) is satisfied with $\delta$ small enough equation (1.3) is also uniformly asymptotically stable.

If equation (1.1) is uniformly stable, i.e., $\alpha=0$ in (2.4) and condition (2.10) is valid, then equation (1.3) is also uniformly stable.

The goal of the following considerations is to obtain estimates for $Q(t, s)$ in which instead of the integral and the sum of the norms of $B(\tau)$ and $B_{k}$ the norm of the following function should enter

$$
D(s)=\int_{s}^{t} B(\tau) d \tau+\sum_{s \leq \tau_{k}<t} B_{k} \quad(s, t \in J, s \leq t) .
$$

We shall note that $D(s)$ is continuous for $s \neq \tau_{k}, D\left(t^{-}\right)=0$ and $D\left(\tau_{k}^{-}\right)=D\left(\tau_{k}\right)=D\left(\tau_{k}^{+}\right)+B_{k}$. Let $y(t)$ be an arbitrary solution of (1.3). From (2.1), taking into account that 


$$
\begin{aligned}
& W\left(t, t^{-}\right)-W(t, s)=\int_{s}^{t} \frac{\partial W}{\partial \tau}(t, \tau) D(\tau) y(\tau) d \tau+\int_{s}^{t} W(t, s) D^{\prime}(\tau) y(\tau) d \tau+\int_{s}^{t} W(t, s) D(\tau) y^{\prime}(\tau) d \tau \\
&+\sum_{s \leq \tau_{k}<t^{2}}\left[W\left(t, \tau_{k}^{+}\right) D\left(\tau_{k}^{+}\right) y\left(\tau_{k}^{+}\right)-W\left(t, \tau_{k}^{-}\right) D\left(\tau_{k}^{-}\right) y\left(\tau_{k}^{-}\right)\right] ; \\
& \frac{\partial W}{\partial t}(t, \tau)=-W(t, \tau) A(\tau), y^{\prime}(\tau)=[A(\tau)+B(\tau)] y(\tau)
\end{aligned}
$$

and

$$
\begin{aligned}
W\left(t, \tau_{k}^{+}\right) D\left(\tau_{k}^{+}\right) y\left(\tau_{k}^{+}\right)-W\left(t, \tau_{k}^{-}\right) D\left(\tau_{k}^{-}\right) y\left(\tau_{k}^{-}\right)+W\left(t, \tau_{k}^{+}\right) B_{k} y\left(\tau_{k}\right) \\
=W\left(t, \tau_{k}^{+}\right)\left[D\left(\tau_{k}^{+}\right)\left(E+A_{k}+B_{k}\right)-\left(E+A_{k}\right) D\left(\tau_{k}^{-}\right)+B_{k}\right] y\left(\tau_{k}\right) \\
=W\left(t, \tau_{k}^{+}\right)\left[D\left(\tau_{k}^{+}\right)\left(A_{k}+B_{k}\right)-A_{k} D\left(\tau_{k}^{-}\right)\right] y\left(\tau_{k}\right)
\end{aligned}
$$

we obtain that

$$
\begin{aligned}
y(t)=W(t, s)[E+D(s)] y(s) & +\int_{s}^{t} W(t, s)[D(\tau)(A(\tau)+B(\tau))-A(\tau) D(\tau)] y(\tau) d \tau \\
& +\sum_{s \leq \tau_{k}<t} W\left(t, \tau_{k}^{+}\right)\left[D\left(\tau_{k}^{+}\right)\left(A_{k}+B_{k}\right)-A_{k} D\left(\tau_{k}^{-}\right)\right] y\left(\tau_{k}\right) .
\end{aligned}
$$

If $W(t, s)$ satisfies estimate (1.2) and there exist constants $M \geq 0, m \geq 0$ and $\eta \geq 0$ such that

$$
|A(t)| \leq M,|B(t)| \leq M,\left|A_{k}\right| \leq m,\left|B_{k}\right| \leq m \quad\left(t, \tau_{k} \in J\right)
$$

and

$$
\left|\int_{s}^{t} B(\tau) d \tau+\sum_{s \leq \tau_{k}<t} B_{k}\right| \leq \eta \quad(s \leq t)
$$

then from (2.12) we obtain that

$$
|y(t)| \leq \varphi(t) \psi(s)(1+\eta)|y(s)|+\int_{s}^{t} \varphi(t) \psi(\tau) \cdot 3 M \eta|y(\tau)| d \tau+\sum_{s \leq \tau_{k}<t} \varphi(t) \psi\left(\tau_{k}\right) \cdot 3 m \eta\left|y\left(\tau_{k}\right)\right|
$$

and by Lemma 1.1 we obtain that

$$
|y(t)| \leq|y(s)| N(t, s) \quad(s, t \in J, s \leq t),
$$

where

$$
N(t, s)=(1+\eta) \varphi(t) \psi(s) \prod_{s \leq \tau_{k}<t}\left(1+3 m \eta \varphi\left(r_{k}\right) \psi\left(\tau_{k}\right)\right) \exp \left(\int_{s}^{t} 3 M \eta \varphi(\tau) \psi(\tau) d \tau\right)
$$

From the estimate (2.15) obtained there follows immediately.

THEOREM 2.2. Let the Cauchy matrix $W(t, s)$ of equation (1.1) satisfy estimate (1.2) and let conditions (2.13) and (2.14) hold.

Then the Cauchy matrix $Q(t, s)$ of equation (1.3) satisfies the estimate

$$
|Q(t, s)| \leq N(t, s) \quad(s, t \in J, s \leq t),
$$

where $N(t, s)$ is given by $(2.16)$.

$$
\begin{aligned}
& \text { COROLLARY 2.4. If }|W(t, s)| \leq K e^{\alpha(t-s)} \quad(s, t \in J, s \leq t), \quad \text { then } \\
& \qquad|Q(t, s)| \leq(1+\eta) K e^{\alpha(t-s)} \cdot e^{3 K M \eta(t-s)+\ln (1+3 K m \eta) i[s, t)}
\end{aligned}
$$

for $s, t \in J, s \leq t$. 
Moreover, if condition (2.8) holds, then

$$
|Q(t, s)| \leq(1+\eta)(1+3 K m \eta)^{\varepsilon} K e^{[\alpha+3 K M \eta+q \ln (1+3 K m \eta)](t-s)}
$$

for $s, t \in J, s \leq t$.

COROLLARY 2.5. In the assumptions of Theorem 2.2 let condition (2.14) be replaced by the more general condition

$$
\left|\int_{s}^{t} B(\tau) d \tau+\sum_{s \leq \tau_{k}<t} B_{k}\right| \leq \eta \quad(s, t \in J, s \leq t \leq s+h)
$$

where $h>0$ is a constant. Then $Q(t, s)$ satisfies the estimate

$$
|Q(t, s)| \leq K(1+\eta) \exp \left\{\left[\alpha+3 K M \eta+\frac{1}{h} \ln (K+K \eta)\right](t-s)+\ln (1+3 K m \eta) i[s, t)\right\}
$$

for $s, t \in J, s \leq t$.

Indeed, estimate (2.20) follows immediately from (2.17) and the fact that the estimate

$$
|y(t)| \leq|y(s)| L^{\exp [\gamma(t-s)+r i[s, t)]} \quad(s \leq t \leq s+h)
$$

implies

$$
|y(t)| \leq|y(s)| L \exp \left[\gamma+\frac{1}{h} \ln L\right)(t-s)+r i[s, t] \quad(s \leq t) .
$$

REMARK 2. In some cases estimate (2.17) is better than estimate (2.7).

EXAMPLE 1. Let equations (1.1) and (1.3) be scalar and $A(t)=-1$,

$$
B(t)=\sin \omega t, A_{k}=1, B_{k}=(-1)^{k} b, \quad 0 \leq b \leq 1, \tau_{k}=\kappa=0,1,2, \cdots, t \in \mathbf{R}_{+} .
$$

Then

$$
|W(t, s)|=e^{-(t-s)+\ln 2 i[s, t)} \leq K e^{\alpha(t-s)} \quad(0 \leq s \leq t),
$$

where $K=2, \alpha=-1+\ell n 2$. In the notation introduced

$$
\delta=1, M=1, m=1, \quad\left|\int_{s}^{t} B(\tau) d \tau+\sum_{\delta \leq \tau_{k}<t} B_{k}\right| \leq \frac{2}{\omega}+b=\eta .
$$

Then $Q(t, s)$ is estimated:

(i) by estimate (2.7)

$$
|Q(t, s)| \leq K e^{\alpha(t-s)} \cdot e^{2(t-s)+\ln (1+2) i[s, t)}
$$

(ii) by estimate $(2.17)$

$$
|Q(t, s)| \leq(1+\eta) K e^{\alpha(t-s)} \cdot e^{6 \eta(t-s)+\ln (1+6 \eta) i[s, t)}
$$

Estimate (2.22) is better than estimate $(2.21)$ if $6 \eta<2$, i.e., if $\frac{2}{\omega}+b<\frac{1}{3}$ which is fulfilled for large $\omega$ and small $b$.

ACKNOWLEDGEMENT. The present investigation is supported by the Bulgarian Ministry of Science and Higher Education under Grant MM-7. 


\section{REFERENCES}

1. BAINOV, D.D. \& SIMEONOV, P.S., Systems with Impulse Effect: Stability, Theory and Applications, Ellis Horwood Limited, (1989).

2. SAMOILENKO, A.M. \& PERESTYUK, N.A., Stability of the solutions of differential equations with impulse effect, Differ. Eqns. 13 (11) (1981-1992)(in Russian). 


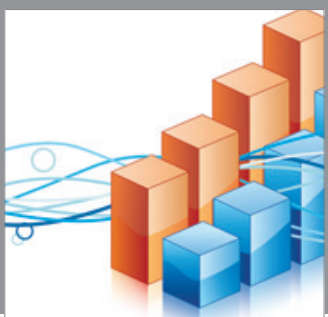

Advances in

Operations Research

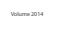

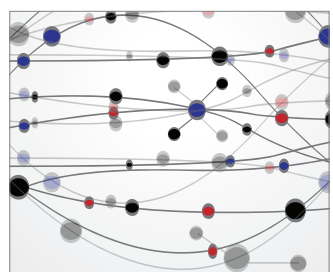

\section{The Scientific} World Journal
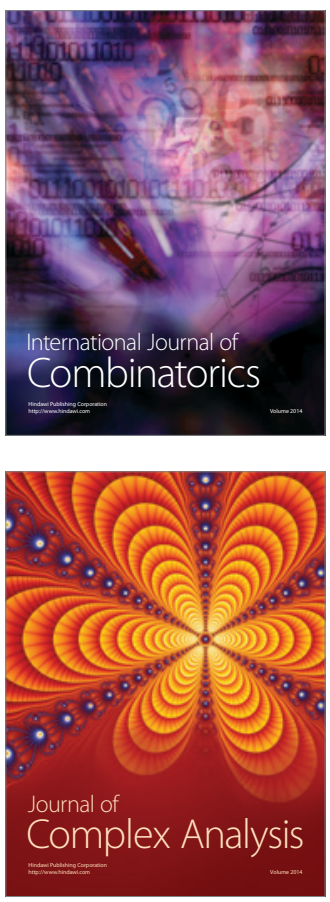

International Journal of

Mathematics and

Mathematical

Sciences
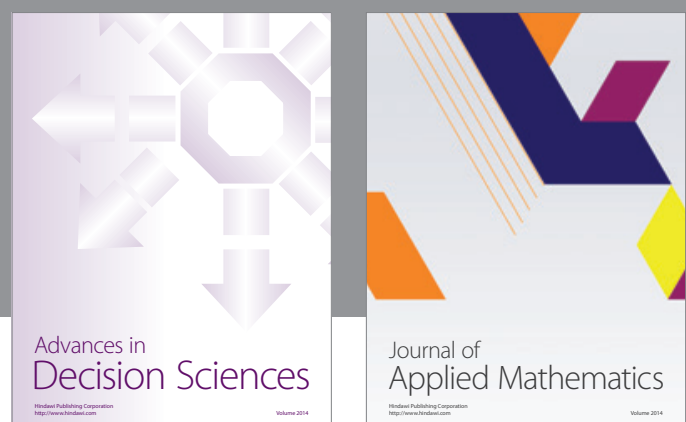

Journal of

Applied Mathematics
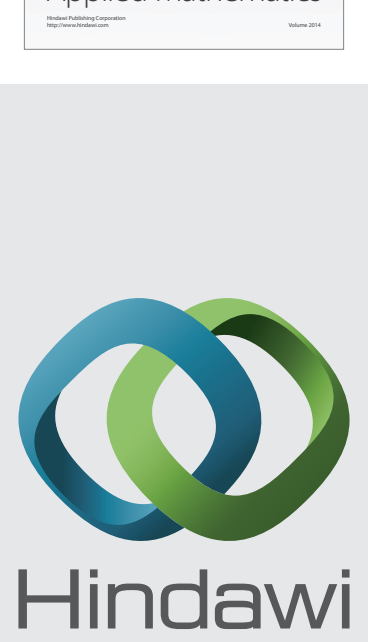

Submit your manuscripts at http://www.hindawi.com
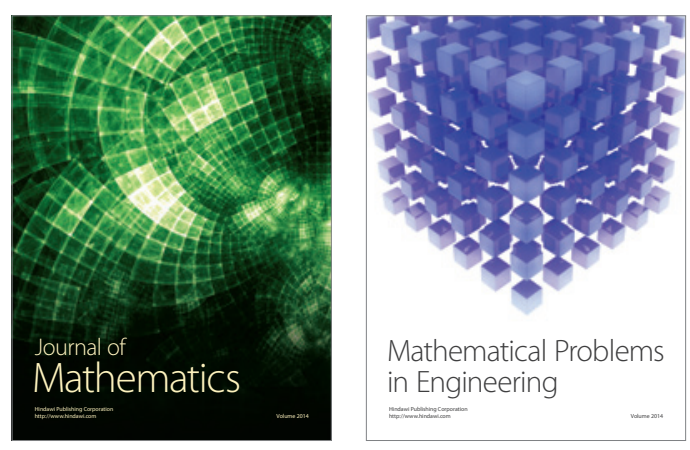

Mathematical Problems in Engineering
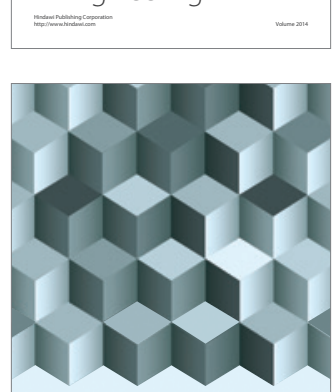

Journal of

Function Spaces
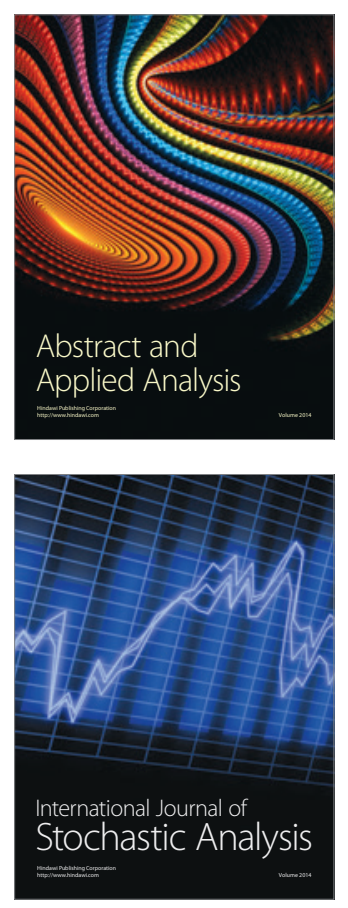

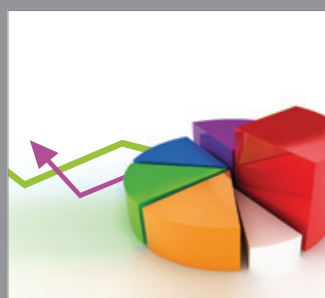

ournal of

Probability and Statistics

Promensencen
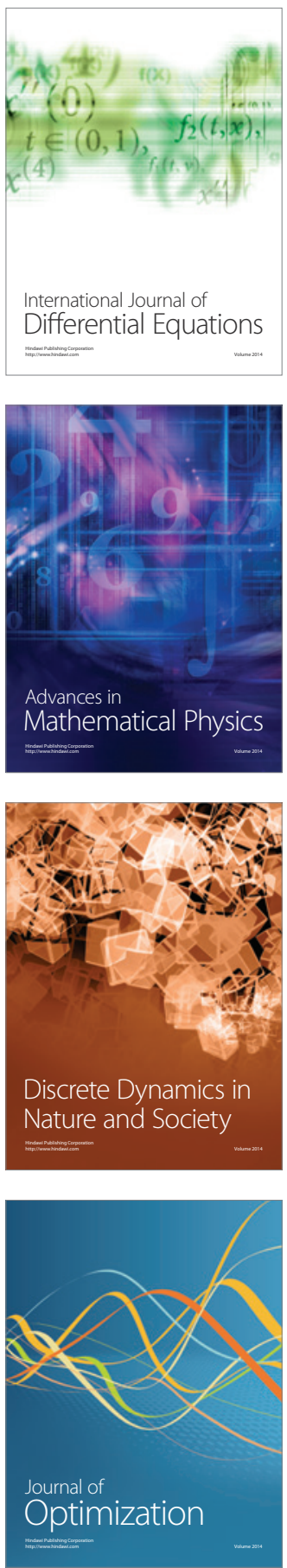